\title{
La représentation des communautés musulmanes auprès des Institutions européennes
}

Joe Carew

\begin{abstract}
Keywords: Religion, Islam, Representation of minorities, Participative democracy.

At least 12 milion muslims are permanent residents on European soil, and Islam must be considered part of the European reality. Two paneuropean muslim organisations - the Federation of Islamic Organisations in Europe (FOIE) and the Forum of European Muslim Youth and Student Organisations (Femyso) - maintain regular relations with the European institutions. This article investigates the capacity of these organisations to represent the heterogenous communities commonly concieved of as "muslim", and on the capacity or the will of the EU institutions to take their contribution on board. It broaches questions such as what is understood by the adjective "muslim" when we talk about society and culture in Europe; the conditions of federation and representation of adherents to these organisations; and the EUs potential to open itself to dialogue with different branches of civil society. The article is based upon a 150 page master thesis written in 2008.
\end{abstract}

Mots-clés : Religion, islam, représentation de minorité, démocratie participative.

Au moins 12 millions de musulmans résident de manière permanente sur le sol européen faisant de l'islam un fait européen. Deux organisations musulmanes paneuropéennes - la Fédération des organisations islamiques en Europe (FOIE) et le Forum of European Muslim Youth and Student Organisations (Femyso) - maintiennent des rapports réguliers avec les différentes institutions de l'Union. Cet article analyse d'une part la capacité de ces organisations de représenter les différentes communautés musulmanes très hétérogènes ainsi que, d'autre part, la capacité ou la volonté des institutions européennes d'intégrer la contribution de ces organisations. L'article est tiré d'un mémoire de 150 pages rédigé en 2008 par l'auteur.

\section{Introduction}

Deux organisations musulmanes paneuropéennes maintiennent des rapports réguliers avec les différentes institutions de l'Union européenne. Il s'agit de la Fédération des organisations islamiques en Europe (FOIE) et du Forum of European Muslim Youth and Student Organisations (Femyso). Tout en prônant une identité musulmane séculaire, ces or- 
ganisations - qui sont les porte-paroles de facto des musulmans auprès de l'UE - cherchent à contribuer à la culture démocratique de l'Union en représentant les intérêts des communautés dont elles sont issues. Dans cet article, nous voulons montrer comment la construction sociale de l'islam et des musulmans qui s'opère dans les débats au sein des institutions européennes influe sur la capacité revendicative des musulmans et contribue à perpétuer certaines difficultés de compréhension liées à leur présence en Europe. Avec la présence permanente d'au moins douze millions de musulmans sur le sol européen, l'islam est dorénavant un fait européen (Allievi 2003 : 451). Et pourtant dans les sphères politiques européennes cette religion est toujours considérée comme un phénomène étranger, ce qui ne facilite pas l'intégration de ces communautés qui représentent une évolution démographique importante en Europe. En même temps l'islam lui-même est en évolution au niveau mondial, en tant que doctrine religieuse et en tant que phénomène social.

Une question qui domine le débat occidental sur l'islam est celle de savoir s'il est compatible avec la laïcité, comprise comme une séparation de la religion et l'État, souvent considérée comme un fondement crucial d'un système démocratique. Le spectre d'un islam fondamentaliste - très médiatisé, mais en réalité très minoritaire - nourrit la crainte occidentale d'un islam inconciliable avec les «valeurs de l'Occident». Certains ont voulu contredire cette thèse en se focalisant sur le potentiel démocratique de l'islam. Ainsi, Hefner montre que le pessimisme occidental se fond sur une myopie à la fois historique et géographique. Il souligne que, même si certains évoquent l'idée de l'islam comme religion et État (din wa dawla), il n'en reste pas moins que les courants réformateurs actuels bénéficient d'une longue histoire de pluralisme et d'une ouverture aux valeurs démocratiques qui débutent à la fin du XIX ${ }^{\mathrm{e}}$ siècle. En effet, il suggère que le véritable « choc des civilisations » vis-à-vis du monde musulman est un choc interne, un conflit entre des visions rivales, telle est l'hétérogénéité de la pensée musulmane aujourd'hui (Hefner 2001 : 493).

Qu'entend-on par «islam européen»? S'agit-il d'un nouveau courant religieux ou d'un véhicule identitaire pour des populations déracinées? Un problème se pose lorsque l'on parle de populations musulmanes en Europe : celui de distinguer les personnes de confession islamique et celles qui sont simplement de "culture musulmane ». Les statistiques sont pour la plupart recensées selon l'origine ethnique et non selon la croyance ou la pratique religieuse. D'après Roy, les enfants d'immigrés arabes en Europe se qualifient plus facilement de «musulman » que d'arabe, ce qui permet de tisser des liens interethniques entre communautés d'origines diverses implantées dans les grandes villes de l'Europe (Roy 1999). Maréchal note que parmi les jeunes musulmans environ 80\% n'ont guère de pratiques religieuses (Maréchal 2001 : 26). En dehors de son contenu spirituel, l'islam participe à l'expression d'une appartenance à un groupe dont les membres tirent force et fierté. Soysal ainsi a pu constater que «l'islam émerge comme un discours sur la participation, et agit comme un outil symbolique et organisationnel pour créer de nouvelles solidarités de groupe [...]. Sans voir de contradiction 
entre l'islam et les valeurs laïques, on mobilise son identité comme une expression politique» (Soysal 1997 : 513-516). Les autorités religieuses sont les gardiens d'un certain patrimoine de valeurs, de rites qui jalonnent les vies et qui ont marqué des structures sociales. En Europe, où les communautés dites «musulmanes» sont presque toujours minoritaires, une personne ou une organisation qui se qualifie de musulmane n'affirme pas forcément une croyance religieuse, mais peut faire appel à une identité partagée.

Encadré $\mathrm{n}^{\circ} 1$ : Profil de la FOIE. Sites internet : www.fioe.org et www.euromuslim.com

Organisation fondée en 1989, enregistrée juridiquement en France, siège à Bruxelles depuis 2007. Compte des membres dans vingt-huit pays, dont neuf qui n'appartiennent pas à l'UE. Elle est ainsi la plus grande organisation musulmane sur la scène publique européenne.

Son assemblée générale est convoquée tous les quatre ans. Elle choisit un président, qui va proposer un bureau exécutif. Une fois validé ce bureau exécutif jouit d'un mandat de 4 ans. Il comporte les services Europe de l'Est, Femmes, Jeunesse et étudiants, Éducation et da'wah, Médias, Finance, Politique, Relations publiques et Secrétariat général.

Bien que la religion joue un rôle central dans la vision de la FOIE, elle se distingue des organisations comme le KEK, le COMECE et la ECR (les principales organisations protestante, catholique et juive) dans le sens où elle est composée essentiellement de personnes de formation séculaire. «Dans le travail - affirme le responsable du service jeunesse - ce n'est pas la spiritualité mais les intérêts des citoyens qui comptent ${ }^{1} »$.

Le rapprochement avec l'UE est un objectif central de la FOIE qui nait d'une « prise de conscience de l'importance du travail qui peut se faire ici à Bruxelles ${ }^{2}$ ». Il nous semble que les buts principaux de la FOIE sont triples: de favoriser l'intégration d'une vision musulmane qui soit compatible avec les principes de la laïcité, d'ouvrir la voie à une plus forte participation musulmane dans les structures des sociétés civiles européenne et nationales et d'aller vers un aggiornamento musulman qui mette la tradition doctrinale en adéquation avec le contexte européen.

${ }^{1}$ Entretien réalisé à Bruxelles le 27/3/08.

${ }^{2}$ Entretien avec le responsable des rapports avec l’EU, réalisé à Bruxelles le 2/4/08. 
Les organisations musulmanes en Europe se distinguent de leurs homologues de confessions autochtones dans le sens où elles sont souvent les principales - parfois les seules - organisations qui regroupent et s'occupent des communautés dont elles sont issues. Rappelons que ces communautés sont souvent marginalisées, caractérisées par une faible participation dans la société civile, un désavantage économique et qui, depuis plusieurs années, sont impliquées dans un certain nombre de problèmes sociaux ou politiques. Ces organisations représentent donc un point d'entrée logique, à défaut d'autres interlocuteurs, pour entamer un dialogue avec les musulmans d'Europe compris dans le sens large d' « origine et de culture musulmanes ».

L'importance de l'immigration dans la présence musulmane européenne introduit forcément des logiques sociologiques nouvelles. Nous ne devons donc pas considérer ces organisations comme des simples organisations religieuses, ni comme de simples groupes d’intérêt communautaires. En réalité elles combinent les deux éléments.

\section{Encadré ${ }^{\circ} 2$. Profil du Femyso - Site internet : www.femyso.net}

Fondé en 1996, siège à Bruxelles. Une assemblée générale des trente-neuf organisations membres (tirées de vingt-deux États-membres de l'UE) se réunit tous les deux ans pour élire un nouveau président et un nouveau comité exécutif (Exco) de 14 personnes, avec un mandat de deux ans. Ce comité est assisté par le «Board of Trustees » composé d'anciens membres de l'Exco.

L'Exco comporte six services : Institutions européennes et ONGs, Échange, Droits de l'homme et islamophobie, Éducation et formation, Communication et relations publiques et Finances. À l'exception du secrétaire général et de la directrice de bureau, les membres de l'Exco travaillent de manière bénévole pour le Femyso et cumulent ce travail avec leur travail associatif dans leurs pays.

L'organisation ne cherche pas à définir un agenda idéologique à imposer à ses membres, mais plutôt d'être un réseau entre les différentes organisations pour qu'elles évoluent ensemble. Elle veut répondre au « besoin important de représentation des musulmans dans les structures européennes ${ }^{3} »$ notamment sur les thèmes de citoyenneté, de discrimination et de droits de l'homme.

Le Femyso ne met en avant aucune vision définie de l'islam. Comme dit son secrétaire général : «On n’est pas une organisation religieuse, on ne fait pas de caté-

\footnotetext{
${ }^{3}$ Femyso description générale disponible sur le site www.femyso.net : 28/11/07.
} 
chisme. Nous on est là pour la politique de la jeunesse [...]. Moi, je dirai que Femyso réunit des valeurs et on les partage. Peu importe la religiosité. Mais c'est vrai que pour adhérer au Femyso il faut être musulman ${ }^{4}$ ». Elle souligne d'ailleurs qu'être musulman c'est seulement « une de nos casquettes »- les jeunes musulmans ont des identités plurielles comme tout le monde.

Comme nous l'avons noté plus haut, une organisation musulmane en Europe ne fait pas forcément appel à la religion, alors qu'une organisation qui se qualifie comme chrétienne aura plus vraisemblablement un contenu religieux explicite. En revanche, ces organisations représentent pour leurs communautés un point focal, un repère qui puise dans un répertoire culturel et identitaire partagé et qui renvoie à la situation particulière des communautés musulmanes dans les pays de l'UE. La mosquée ou l'organisation musulmane ne jouent pas un rôle analogue à celui du lieu de culte ou de l'organisation religieuse autochtone dans la mesure où elles ont souvent plus de poids symbolique ou pratique dans la vie de la communauté.

\section{L'Union européenne et les Religions}

Certains se demanderont pourquoi l'UE, un pouvoir public (donc séculier) devrait maintenir des rapports aves des groupes religieux. La sphère publique européenne n'est elle pas censée être laïque ? La religion n'est en réalité jamais sortie de cette sphère publique, dans le sens où de nombreux acteurs agissant en son sein sont motivés par leurs croyances religieuses. D'ailleurs des liens entre les pouvoirs publics et les autorités, bien que souvent informels, existent bel et bien, et donc leur absence des processus décisionnels officiels n'empêche nullement leur influence sur le gouvernement (Casanova 2001 : 1041-1080). Du côté du pouvoir public, la coopération avec les groupes religieux peut également être avantageuse : ce sont parfois des acteurs sociaux importants, disposant de ressources financières et de réseaux de contacts utiles. Dans certains cas, leur implantation internationale les rend intéressants au niveau des Affaires étrangères. Dans le cas de l'islam, de nombreux États européens cherchent actuellement à mettre sur pied une instance représentative des communautés musulmanes afin d'avoir un interlocuteur sur les enjeux les concernant.

Mais peut-on légitimer la présence de voix religieuses dans le débat politique, alors que celui-ci est censé être fondé sur la rationalité de l'argumentation qui s'y exprime? Puisque les frontières du public et du privé sont floues, on peut postuler qu'en interdi-

${ }^{4}$ Entretien réalisé à Bruxelles le 28/02/08. 
sant l'expression de certaines idées, on risque de réduire la capacité démocratique de la sphère publique. Il est nécessaire d'examiner les arguments sur le plan qualitatif, pas simplement de les exclure parce que c'est un religieux qui les expose. La source ou la motivation de l'argumentation est moins importante que son contenu et l'idiome dans lequel il s'exprime.

Habermas postule que, dans l'idéal, la sphère publique devrait être l'instrument à travers lequel tout membre de la société peut contribuer à la formulation et l'adoption de toute décision politique collective et de normes sociales générales (Herbert Op. cit. p.100). Ce n'est pas donc la neutralité du discours qui importe, mais l'égale opportunité pour tout citoyen d'initier et de continuer la communication. Par exemple, si l'on propose d'interdire le mariage homosexuel puisqu'il représente une abomination aux yeux de dieu, on exclut ceux qui ne croient pas en dieu de la possibilité de répondre à la proposition dans les termes qu'elle établit. Si par contre, tout en se motivant de ses croyances religieuses, on propose cette même interdiction en faisant appel à une prétendue menace pour la famille nucléaire, le débat est accessible à tous, dans le mesure où il relève d'un enjeu concret, non pas de la foi individuelle.

L'UE, quant à elle, a été décrite comme étant « church blind» (Jansen 2000 : 103) puisqu'avant la rédaction du Traité constitutionnel elle s'est défendue d'aborder la question de la religion ${ }^{5}$. Deux facteurs informent de cette «church blindness ». D'abord la tradition européenne du sécularisme fait que la question de la participation des religieux dans des processus politique est pour le moins épineuse. Davie note que « les hommes politiques de l'Europe occidentale répugnent à faire un usage politique des références religieuses » (Massignon 2007 : 12). Ensuite, les énormes différences dans les modèles nationaux de rapports entre religions et pouvoirs publics ne facilitent pas l'accord sur les dynamiques des relations que l'UE devrait maintenir avec des acteurs religieux. En l'absence d'un consensus « le silence est apparu comme la seule réponse possible » (Foret $2005: 6$ ).

Ainsi les liens entre l'UE et les religions sont restés strictement informels. Mais cela ne signifie pas que l'influence de ces acteurs est forcément restée mineure. Pourtant le champ des lobbys religieux à Bruxelles fait preuve d'une forte hétérogénéité quant aux ressources dont les acteurs disposent et aux démarches qu'ils poursuivent. Les catholiques sont de loin les mieux dotés et les plus investis. Avec les protestants (pourtant beaucoup plus divisés) ils dominent la scène au point où les autres confessions se voient éclipsées (Schlesinger et Foret 2006 : 68). Les deux organisations musulmanes qui sont les interlocuteurs officiels de la Commission ont en effet souvent du mal à se faire entendre, comme nous allons le montrer dans ce qui suit.

\footnotetext{
${ }^{5}$ L'article 52 de ce Traité (devenu l'article 17 du Traité de Lisbonne) devait établir un dialogue « ouvert, transparent et régulier » avec les religions au nom de la démocratie participative.
} 
La majeure partie du lobbying religieux se fait avec les outils habituels des groupes d'intérêt. Il s'agit de tisser des liens avec les décideurs, d'essayer de créer des réseaux sociaux par le biais de conférences et d'autres rencontres, de se regrouper autour d'enjeux ou de buts précis. Plusieurs facteurs peuvent favoriser la réussite de la démarche : une bonne connaissance des structures organisationnelles du pouvoir public et des rapports de confiance et d'amitié de ceux qui l'incarnent, des ressources humaines suffisantes, pour tout cela les ressources financières sont d'une importance capitale. Les principaux points d'accès des deux organisations étudiées sont doubles. À la Commission, un conseiller auprès du Président est hébergé par le $\mathrm{BEPA}^{6}$; des contacts ponctuels existent également avec certains directorats généraux. Au Parlement, ces organisations semblent avoir plus de difficultés à s'imposer, même si une poignée d'eurodéputés sont à leur écoute.

\section{La représentation des intérêts musulmans auprès de la Commission}

Depuis le début des années 1980 la présidence de la Commission a trouvé opportun d'entretenir un dialogue centralisé, plus ou moins multilatéral, avec des groupes religieux et humanistes. Au cours des 25 dernières années, ce dialogue est passé par plusieurs étapes, selon l'importance que le président du moment y attachait, et selon le style des différents responsables, qui jouissent d'une remarquable liberté quant à leurs méthodes. L'inclusion de voix musulmanes dans ce dialogue a été tardive : leur première participation date de 1994, à la toute fin de la période Delors. Elles arrivent donc dans une situation où les paramètres de l'échange sont déjà établis.

L'initiative pour la création de ce poste ne venait pas de la présidence, mais de l'homme qui allait l'occuper, Umberto Stefani (Massignon 2007: 112). Notons, d'ailleurs, le caractère presque honorifique du poste de Stefani, un fonctionnaire en retraite qui n'était pas rémunéré par la Commission, et qui y travaillait à temps partiel. Massignon remarque que "par ses convictions et son réseau de relations, Stefani privilégiait les relations avec l'Eglise catholique prise dans son sens le plus hiérarchique : il se disait lui-même « homme de foi et de papauté » [...] il avait reçu une distinction honorifique du Saint-Siège et on le disait proche de l'Opus Dei. » (Massignon 2007 : 113). Si l'on s'attend à une certaine neutralité dans la nomination à ce poste qui relève d'une matière politiquement sensible, le choix d'un catholique très dévot et proche du Vatican peut paraitre étrange. Ce type de considération ne semble cependant pas avoir troublé

\footnotetext{
${ }^{6}$ Bureau des conseillers de politique européenne, vous trouverez les informations relatives à cet
} organe notamment à l'adresse suivante : http://ec.europa.eu/dgs/policy_advisers/index_en.htm 
les présidents successifs de la Commission. En effet, jusqu’à présent tous les conseillers ont été plus ou moins proches d'une Eglise.

Les liens entre la Commission et ses interlocuteurs religieux étaient d'abord ponctuels et surtout bilatéraux. Sous la présidence Delors, le rythme du dialogue commence à s'accélérer. Delors disait : « on ne tombe pas amoureux d'un grand marché » et pour lui les religions et les groupes humanistes étaient des partenaires privilégiés dans la recherche de la substance affective à laquelle les européens pouvaient s'attacher, «l'âme de l'Europe ». C'est sous Delors, en 1994, qu'une présence musulmane - pourtant plutôt symbolique - est enfin invité à ce dialogue du BEPA (alors dénommé la Cellule de prospective). Au début les invités musulmans étaient des personnalités comme des intellectuels ou des cheiks, non pas des organisations. Ce n'est qu'en 1999 qu'une petite organisation, peu identifiable, le CMCE, se fait inviter, et il faut attendre 2003 pour qu'une organisation conséquente et engagée comme le Femyso y soit impliquée.

Fort de l'élan de la période Delors, ce dialogue atteint le zénith de son utilité comme focalisation du lobbying des groupes religieux sous la présidence de Santer. À ce moment, il est composé de trois cercles concentriques de dialogue. Un premier cercle n'inclut que des groupes catholiques, protestants et orthodoxes, qui participent à des séminaires de dialogue prospectifs. Un deuxième cercle de dialogue se réunit moins régulièrement ; il inclut des juifs, des humanistes et des musulmans. Le dernier cercle, ouvert à tous, consiste à des réunions de briefing suite aux sommets européens. Ces réunions ont un caractère principalement informatif plus que d'échange. Or, pendant les périodes Prodi et Barroso, les deux premiers cercles, les seuls véritables lieux de dialogue, sont démantelés, laissant seulement place aux briefings bisannuels. Les contacts sont maintenant plus ponctuels, et souvent à l'initiative des groupes religieux eux-mêmes. Ainsi les partenaires les plus impliqués et les mieux dotés semblent perdent leur intérêt pour ce Dialogue officiel. Comme le souligne Massignon, « le cœur de l'engagement des Églises n'est plus à ce niveau transversal et politique, mais dans les contacts informels c'est-à-dire dans le lobbying auprès des Directions générales de la Commission » (Massignon $2007:$ 172).

Quel peut donc être actuellement le but de ce dialogue ? Parmi les trois principaux buts qu'il nous cite, le responsable actuel, Jorge César das Neves, a souligné précisément la nécessité d'engager des voix musulmanes. Il indique, par exemple, que bon nombre d'individus au sein des institutions de l'UE, qui ne voient pas l'intérêt d'un dialogue avec les religions, y deviennent favorables dès lors que l'islam y est aussi représenté .

${ }^{7}$ Entretien réalisé à Bruxelles le 28/02/08. 
En cela l'Union reflète le désir de plusieurs États européens de trouver un interlocuteur musulman pour faciliter les rapports avec ces communautés. Or comme note Frank, les communautés elles-mêmes s'en méfient parfois comme d'une tentative d'interférence (Peter 2006 : 183-212). Pourquoi la Commission manifeste-t-elle ce désir? Selon un fonctionnaire : «la Commission est convaincue que nous devons [...] moderniser certaines communautés qui n'ont pas suivi ces processus d'illumination de la raison et de modernisation, de la séparation de l'église et de l'État. Evidemment, il s'agit principalement des musulmans ». Il est dommage que cette image stéréotypée des musulmans européens perdure au sein des institutions, a fortiori lorsque les deux interlocuteurs officiels musulmans déclarent haut et fort leur engagement en faveur d'une Europe sécularisée.

En effet, si l'on regarde les activités de la FOIE et du Femyso, on voit que ces organisations se focalisent déjà et par elles-mêmes sur des questions qui sont chères aux décideurs européens, que ce soit la participation de tous à une société démocratique, les droits de la femme, l'insertion professionnelle des jeunes, etc. Malgré ces efforts, l'UE semble rester convaincue que les musulmans ont encore pas mal à apprendre. Jorge César das Neves explique qu'il faut instrumentaliser les interlocuteurs religieux pour diffuser un message aux citoyens :

« Si je dis une chose, j’ai une certaine crédibilité, mais si un leader musulman le dit, il a une autre crédibilité... c'est ça le but principal du dialogue interreligieux, de dire, pourquoi vous n'utilisez pas le type de discours que nous utilisons? C'est à vous de parler ... à vos gens, de leur dire de respecter la femme. Ainsi on pourrait passer le message que les religieux, ce ne sont pas des gens dangereux 8 ".

Or, quelque part, l'idée que certains « religieux » sont dangereux semble toujours travailler l'esprit de l'UE.

Il existe d'autres lieux d'incompréhension entre les organisations musulmanes et l'UE. Lors de notre enquête de terrain, il nous est apparu clairement que le manque de ressources financières des organisations musulmanes les empêche de faire mieux connaitre leur message au sein de l'UE. En outre, leur arrivée très récente dans les sphères européennes de dialogue signifie qu'elles n'y ont pas encore développé de réseaux significatifs de contacts. Ces obstacles à la participation ne semblent pas être véritablement compris au sein des institutions. Un fonctionnaire nous a expliqué que, d'un côté, les opportunités de dialoguer avec les chrétiens et les juifs «sont nombreuses, parce que ces gens sont habitués à nous, à nos façons de faire ». Surtout ils sont très actifs - «ils nous bombardent parfois ». De l'autre côté, il trouve que les musulmans sont plus discrets : «apparemment, ils ne se sentent pas portés à interagir avec nous, à nous présenter leurs points de vue, à faire du lobbying comme d'autres le font, qui ont vraiment

${ }^{8}$ Entretien réalisé à Bruxelles le 28/02/08. 
compris... Je n'ai pas le sentiment qu'ils veulent influer sur le programme de la Commission. »

Nos interviewés au sein de la FOIE et du Femyso ont rejeté de manière véhémente l'idée que leurs organisations manquaient de volonté de participer. Outre les ressources financières et humaines limitées de ces organisations, d'autres facteurs entravent leur percée sur la scène politique européenne. L'absence d'organisation centralisée et hiérarchisée dans l'islam sunnite (majoritaire en Europe) peut poser des obstacles organisationnels dans le sens où il n'y existe pas de tradition de se fédérer tous sous une même bannière. Ceci explique en partie la voix faible et divisée des musulmans d'Europe (voir par exemple Peter 2006 ou Dassetto 2001). D’ailleurs, comme nous verrons par la suite, la tradition séculaire européenne dresse une certaine barrière à la participation de tous ceux qui sont perçus comme des « religieux », et que les musulmans - minoritaires, récemment implantés et faibles au niveau socioéconomique - ont plus de mal à franchir que d'autres.

Enfin, la citation ci-dessus allègue une autre raison aux rapports assez peu fructueux entre l'UE et les musulmans : Ces derniers ne sont pas «habitués à nous, à nos façons de faire ». Les structures de dialogue se sont développées sans la participation des musulmans, et les acteurs européens semblent les percevoir comme extérieurs au système, sinon à l'Europe. Cela ne facilite pas leur intégration dans ce dialogue. Mais les musulmans doivent-ils produire l'entièreté de l'effort pour «s'habituer» à l'UE - ou l'UE pourrait-elle montrer plus d'ouverture d'esprit de son côté envers les organisations musulmanes?

\section{Représentation auprès du Parlement européen}

En ce qui concerne le Parlement européen (PE), nous avons pu identifier d'importantes similitudes par rapport aux attitudes observées ou décrites à la Commission. D'abord, le simple fait d'être catégorisé comme des « religieux » produit d'emblée une méfiance ou un désintérêt vis-à-vis des organisations musulmanes chez bien des acteurs européens. Du coup ils ne vont pas au-delà de l'aspect «musulman » pour découvrir quel est en réalité l'agenda social ou politique de ces organisations et pour s'en entretenir avec elles. Lors de notre rencontre avec le conseiller du président de la Commission sur le dialogue avec les religions, celui-ci nous a expliqué :

Au cours de l'histoire, les Églises se sont rendues redondantes ou du moins c'est ce que l'on pense. Personne n'a le temps d'écouter des prêtres. Beaucoup disent : «Je suis désolé, si vous voulez parler des affaires sociales, on a nos propres experts, nous n'avons pas le temps pour ça ».

${ }_{9}^{9}$ Entretien réalisé le 28/2/08. (Nous traduisons de l’anglais). 
Plusieurs représentants des organisations musulmanes nous ont parlé de la difficulté de trouver des personnes prêtes à les écouter au sein des institutions européennes. Ils attribuent ce fait à une méfiance non seulement à l'égard des musulmans, mais de l'ensemble des religieux.

Or, si les lobbys religieux à Bruxelles sont considérés comme « redondants », la question des ressources est encore plus cruciale dans le sens où il faut consacrer du temps et de l'argent simplement pour s'informer des activités et des rencontres qui ont lieu. Une organisation qui est invitée à participer à une rencontre peut en tirer du profit : Elle sera au moins incluse dans la discussion. Or, si les organisations religieuses sont souvent obligées de rechercher les événements et d'acquérir le droit d'y participer par ellesmêmes, l'avantage aux plus riches est énorme. L'organisation bien dotée pourra participer aux rencontres desquelles la première organisation sera simplement exclue. Ainsi, pour surmonter la méfiance ou l'indifférence, les groupes plus riches et mieux organisés bénéficient d'un avantage considérable. Un fonctionnaire nous a expliqué pourquoi il s'occupe plus de certains groupes que d'autres :

Parfois on considère la communauté juive comme une minorité, mais ils ont une organisation puissante ici, soutenue par des milieux influents new yorkais [...]. La COMECE, le KEK, ils sont très actifs ; les organisations musulmanes, il faut que moi j’aille à leur recherche.

On voit donc que ce manque de ressources se traduit par un accès plus limité, et par une impression de désintérêt perçue par les institutions européennes qui ne facilitent évidemment pas l'inclusion aux processus de dialogue ou d'échange.

Pour revenir à l'idée de la méfiance des groupes religieux, elle parait être assez courante chez certains eurodéputés : les groupes religieux eux-mêmes sont les premiers à le constater (Charentenay 2001) et plusieurs des eurodéputés que nous avons contactés ont en effet refusé de nous parler de religion. Les différentes approches nationales semblent influer sur les attitudes. En Grande-Bretagne, il existe une religion d'État, et des hauts prélats de l'Église anglicane siègent même dans le parlement et cependant il n'est pas question d'une fermeture vis-à-vis des autres religions. Selon une représentante de la FOIE, d'origine européenne, cette culture de pluralisme ouvert trouve son reflet dans le fait que la plupart des députés qui acceptent de dialoguer avec la FOIE sont des Britanniques. Elle constate «une nette différence» par rapport à la culture française, où la doctrine de la laïcité représente, semble-t-il, un mur infranchissable «parler aux français, ce n'est même pas possible, on parle chinois ».

La représentation auprès du PE des intérêts des communautés musulmanes se voit entravée lorsqu'elle se fait par le biais d'organisations caractérisées comme religieuses, en raison de cette barrière à leur accès qu'est la conception que certains se font de la laïcité. Lors d'une brève rencontre avec l'eurodéputée française Tokia Saïfi, où nous abordions des questions de minorités ethniques et de cohésion sociale dans un contexte postcolonial, celle-ci s'est montrée incapable (ou peu encline) à dépasser le thème global de l'islam qui encadrait notre recherche. Nous lui avons suggéré qu'une députée issue d'immigration maghrébine pourrait être bien placée pour faciliter un dialogue entre ces 
communautés et les pouvoirs publics; elle a répondu par un discours mettant en valeur la doctrine républicaine selon laquelle elle est députée européenne et française, point final. Elle n'avait jamais pensé qu'il pourrait être utile de collaborer avec d'autres élus issus de ces mêmes populations pour faciliter leur insertion sociale. Elle ramenait souvent la discussion sur la question de l'islam pour dire qu'elle n'avait rien à dire là-dessus : «Ce n'est pas une question qui m'occupe ${ }^{10} »$.

Il va de soi qu'elle est libre d'avoir ou non des opinions sur les communautés musulmanes d'Europe. Pourtant, avant son élection au PE en 1999, elle était l'organisatrice d'activités de dialogue culturel et spirituel entre les trois religions monothéistes et coordinatrice d'un mouvement national en faveur d'une laïcité ouverte. Aussi peut-on imaginer qu'elle avait déjà abordé le thème de l'intégration des communautés musulmanes (compris dans le sens socioculturel, et non strictement religieux) dans les systèmes politiques européens. Se serait-elle donc tue par le fait que selon sa vision de la laïcité un député ne devrait pas maintenir un intérêt pour des questions ou des organisations religieuses?

Nonobstant tout ceci, la religion est loin d'être absente du Parlement, ni des débats dans l'hémicycle, ni des réseaux qui se tissent entre députés dans les coulisses. Nombreux sont les discours que des papes, des patriarches, des évêques et des rabbins ont adressés à l'assemblée au fil des années. Le thème de la religion a dominé l'année du dialogue interculturel en 2008 à tel point que la députée britannique Sarah Ludford a pu dire :

Le président ne doit pas permettre la dominance de la liste des intervenants par des personnages religieux. C'est l'année du dialogue interculturel, non interreligieux. Je tiens à dire également que nous ne devons pas faire de la religion un synonyme de la culture $^{11}$.

D'ailleurs le catholicisme et le protestantisme ne sont pas les seuls à être impliqués. Depuis quelques années le parti populaire européen (PPE) organise une conférence avec l'Eglise orthodoxe sur des questions de cohésion sociale en Europe de l'est. D'ailleurs le Parlement n'est pas sans vouloir mieux connaittre le rôle positif que peuvent jouer les acteurs religieux dans la diffusion du message européen et même d'approfondir la compréhension de la présence musulmane sur son territoire: une étude en deux volumes intitulée Convergences musulmanes, a été réalisée sur les recommandations du PE (Massignon 2007 : 202).

Nous devons donc constater que l'attitude du PE envers la religion est plus nuancée qu'une simple méfiance aboutissant à une volonté d'écarter les religieux. En même

\footnotetext{
${ }^{10}$ Entretien réalisé à Strasbourg le 20/3/08.

11 www.sarahludfordmep.org.uk (nous traduisons de l'anglais).
} 
temps, force est de constater que le soutien intra muros de certaines confessions leur donne un avantage considérable par rapport à d'autres. On pense notamment à la pétition montée par l'eurodéputée Elizabeth Montfort du PPE pour une reconnaissance officielle des «origines chrétiennes» de la culture européenne. Massignon constate que des catholiques et des protestants montent des campagnes de lobbying «par la pastorale » (Massignon 2007 : 252), qui consistent en des conférences organisées pour des fonctionnaires et des députés croyants sur des thèmes européens. Sur le même principe des prayer breakfasts organisées par Hans Gert Poettering, président du PE, regroupent des eurodéputés autour d'une sentiment spirituel partagé. Notons qu'aucun député musulman n'y a été invité. Au fil de ce type de rencontre se construisent des communautés de politiques publiques potentielles, bénéfiques aux confessions impliquées. Il ne semble pas que les musulmans aient pu profiter d'une pareille ressource.

À la méfiance des religieux déjà constatée dans certains secteurs des institutions de l'UE, s'ajoute le problème que les européens considèrent souvent l'islam comme un phénomène étranger. Surtout, ils le perçoivent à travers le prisme du Moyen-Orient. Un eurodéputé nous a fait la remarque que «les problèmes que nous avons ici en Europe sont au moins un écho de conflits ailleurs, donc il y a une dimension de politique étrangère ». Cela n'est pas faux. Il y a cependant des raisons de croire que ce sont les européens qui font le plus souvent ce lien avec la politique étrangère, en débattant de cet amalgame occidental qui est «le problème de l'islam». Nous n'avons pas constaté d'expertise particulière, ou de volonté de discuter, des affaires extra-européennes de la part des musulmans interviewés, qui ont souligné par ailleurs que, de plus en plus, le principal attachement que les musulmans en Europe ressentent est pour le pays européen où ils sont nés.

Depuis les années 1990, aux conférences organisées par l'UE, on a souvent invité exclusivement des personnalités des pays arabes pour représenter l'islam. Le Parlement continue cette tradition quelque peu malheureuse : Sarah Ludford a parlé ainsi dans l'hémicycle en janvier 2008 :

Je suis déçue que le grand mufti de Syrie ait été invité au même titre que le pape et le chef rabbin britannique. Il aurait été plus approprié d'inviter un européen [...] et ainsi de transmettre le message que les eurodéputés considèrent l'islam comme une religion mainstream européenne ${ }^{12}$.

Or, en effet, il y a bien raison de croire qu'au sein du Parlement, nombreux sont ceux qui conçoivent l'islam principalement comme un phénomène étranger qui s'est implanté en Europe. Cette approche ne favorise pas particulièrement la démarche des organisations musulmanes européennes. Inviter des musulmans européens essentiellement lorsqu'on veut les faire dialoguer avec des extra-européens, comme dans les conféren-

\footnotetext{
12 www.sarahludfordmep.org.uk (nous traduisons de l'anglais)
} 
ces suscitées, ne témoigne pas d'une volonté de discuter de leurs droits citoyens, ou des problèmes liés à l'intégration des minorités ethniques. Au contraire, il semble focaliser encore leur «islamité » (en tant que caractère extra-européen) et chercher à faire des ponts entre deux civilisations qui sont - comme disent certains - incompatibles. Le Moyen-Orient est certes important dans la vision du monde des musulmans croyants. Or, leur identité ne se résume pas à cela. D'ailleurs, on peut se demander quel est le rôle de la Turquie dans ce débat. Presque autant de musulmans en Europe sont d'origine turque, que d'origine maghrébine (Dassetto 2001: 17), et pourtant il n'en est guère question dans ces débats. Est-il possible que l'islam intéresse moins les parlementaires européens si les liens avec le fondamentalisme et la violence sont moins évidents?

\section{Conclusions}

Les relations entre les organisations musulmanes et le Parlement, tout comme celles avec la Commission, sont entravées par trois facteurs principaux. D'abord, un manque de ressources et d'acteurs à leur écoute. Ensuite, le caractère plus ou moins religieux assigné à ces organisations empêche de voir les enjeux dont ces dernières s'occupent principalement. Une fermeture d'esprit lorsque le mot religion est prononcé ne permet pas à ces organisations de faire valoir leur programme au sein du Parlement, alors que nous avons vu que ce programme se rapproche souvent plus de celui d'un groupe de pression sociale, que de celui d'une Église. Finalement, il y a cette idée qui prévaut chez nombres d'européens selon laquelle l'islam serait un phénomène moyen-oriental, ce qui entrave son acceptation comme « religion mainstream européenne », pour reprendre la phrase de Ludford. On oublie ainsi la contribution de l'islam à la culture européenne, et surtout on ne favorise pas l'émergence d'un dialogue sur les problèmes sociaux spécifiques auxquels les musulmans d'Europe font face aujourd'hui.

En même temps, les organisations musulmanes, elles aussi, semblent avoir raté certaines opportunités. C'est le cas par exemple du European Muslim Forum (EMF) fondé en juin 2005 par les eurodéputés Sarah Ludford et Sajjad Karim dans le but de créer « une porte » par le biais de laquelle des organisations musulmanes à tout niveau pourraient accéder au Parlement ${ }^{13}$. Si hypothétiquement ce forum existe toujours ${ }^{14}$, son cofondateur avoue que sa réussite a connu des limites : en réalité il n'y a eu aucune réponse de la part des musulmans. Pour leur part, le Femyso et la FOIE ont eu connaissance de cette initiative, mais ils n'ont pas su expliquer pourquoi ils n'y participaient pas.

\footnotetext{
${ }^{13}$ Entretien avec Sajjad Karim réalisé à Strasbourg le 11/03/08.

14 Sur le site web de $\mathrm{M}$ Karim on apprend qu'il en est toujours le président. www.sajjadkarim.eu/index.php?sectionid=104.
} 
Des deux côtés il reste des progrès à faire pour que cette représentation des intérêts des musulmans s'améliore. Au fil de leurs contacts avec l'UE, les deux organisations musulmanes ont fait preuve d'une capacité de développer et d'affiner leur démarche. Côté UE, il faudra que des fonctionnaires et des députés renoncent à leurs illusions et préconceptions sur ce qu'être musulman veut dire en Europe aujourd'hui. Sans quoi il est difficile d'envisager comment ce dialogue pourrait avancer. La représentation des intérêts minoritaires au sein de l'UE est cruciale surtout pour des questions d'affaires sociales et de l'emploi, de culture et éducation, et de justice et liberté. L'Union saura-t-elle garantir la représentation de ses citoyens en veillant à dialoguer avec toutes sortes d'acteurs de la société civile? En écoutant mieux ses citoyens elle donnerait à ces derniers plus d'opportunité de s'accrocher à l'UE et de se l'approprier. Sur ce point il vaudrait mieux agir tôt que tard.

\section{Bibliographie}

AlLIEVI S. (2003), «The international dimension », In : MARÉCHAL B. et al., Muslims in the enlarged Europe. Religion and Society, Leiden, Brill.

Casanova J. (2001), «Civil Society and Religion: Retrospective Reflections on Catholicism and Prospective Reflections on Islam », Social Research, vol. 68, (4), pp. 1041-1080.

Charentenay P. (2001), « La Charte Européenne et la laïcité », Etudes, nº 9, tome 395, pp. 153-164.

DAvie G. (2001), «L'Europe : l'exception qui confirme la règle ? » In : Berger P., Le réenchantement du monde, Paris, Bayard.

FORET F. (2005), « Dieu est-il soluble dans la gouvernance multi-niveaux ? L'émergence d'une politique publique religieuse européenne, Rapport au congrès national de l'Association Française de Science Politique », Table ronde $n^{\circ} 6$ Villes, régions, États, Europe: l'action publique à l'épreuve des changements d'échelle, Lyon 14-16 septembre 2005.

Hefner R. (2001), «Public Islam and the Problem of Democratisation », Sociology of Religion, 62: 4.

JANSEN T. (2000), «Europe and Religions: the Dialogue between the European Commission and Churches or Religious Communities ", Social Compass, vol 47, $\mathrm{n}^{\circ} 1, \mathrm{p} 103$.

MARECHAL B. (2001), "Appartenances et pratiques», In: DASSETTO F. et al, Convergences musulmanes: Aspects contemporains de l'islam dans l'Europe élargie, Paris, L'Harmattan. 
Massignon B. (2007), Des Dieux et des fonctionnaires, Presses Universitaires de Rennes.

Peter F. (2006), «Rationalités du pouvoir et incorporation de l'Islam », Sociologie et Sociétés, vol 38, $\mathrm{n}^{\circ} 1$, pp 183-212.

ROY O. (1999), Vers un islam européen, Paris, Editions Esprit.

SCHLESINGER P., Foret F. (2006), « Political Roof and Sacred Canopy? Religion and the EU Constitution », European Journal of Social Theory, vol. 9, (1).

SOYSAL Y. (1997), "Changing parameters of citizenship and claims-making: Organised Islam in European Public Spheres », Theory and Society, 26. 